\title{
Gobernanza, redes y territorio
}

\author{
Johannes Glückler¹, Johannes Rehner², Michael Handke³
}

La gobernanza es a la vez un concepto controvertido y una creciente preocupación empírica relacionada con una miríada de desafíos sociales contemporáneos y dilemas de acción colectiva. El propósito de este número especial es invitar a una conversación sobre nuevos conceptos de gobernanza y su relación con las redes y el territorio en los estudios ambientales, regionales y urbanos.

\section{Enmarcando el concepto gobernanza}

Por un lado, la gobernanza se ha convertido en un concepto casi universal y global, el que durante décadas ha atraído el interés de investigadores de muchas disciplinas a la hora de abordar los dilemas de la acción colectiva y facilitar la coordinación eficaz de los intereses y los recursos hacia objetivos comúnmente aceptados. En su sentido más amplio, que incluye la multitud de enfoques, gobernanza denota la coordinación de acción colectiva (Jessop, 1998). Las contribuciones a las teorías y perspectivas de la gobernanza se originan en diversos campos disciplinarios, incluyendo las ciencias políticas (Ostrom, 1990; 2005; Hirst y Thompson, 1996; Crouch, 2005; Feiock, 2007), la sociología (Mayntz y Scharpf, 1995; Jessop, 1998), la conomía (Williamson, 2005), los estudios de organización (Gereffi et al., 2005), geografía (Storper y Harrison, 1991; Liverman, 2004; Hughes, 2006) y estudios de planificación (Nuissl et al. 2012; Lukas, 2019).

Por otra parte, las concepciones de gobernanza varían considerablemente, y sus significados dependen de las perspectivas disciplinarias, las tradiciones teóricas y el enfoque empírico. Manuales recientes ofrecen una visión general de la amplitud interdisciplinaria de ideas y debates (Bache and Flinders, 2004; Cerrillo, 2005; Chhotray y Stoker, 2009; Bevir, 2010; Levi-Faur, 2012; Ansell y Torfing, 2016). La multiplicidad de términos asociados a gobernanza abarca desde campos prácticos como la gobernanza empresarial, contractual, de proyectos, pública, privada o sin fines de lucro, hasta la gobernanza urbana, de suelo, de riesgo, del medio ambiente y del clima. Esta multidisciplinariedad también incluye los enfoques conceptuales o normativos de la gobernanza en red, la gobernanza colaborativa, participativa, justa o buena gobernanza, y a diversas escalas desde la local, regional y nacional, hasta la gobernanza mundial y la gobernanza multinivel.

A veces es útil que un concepto emergente no se defina de manera demasiado específica, para así atraer la atención y el esfuerzo de investigadores con el fin de estimular la conversación académica. Sin embargo, esta diversidad puede dificultar el intercambio, la comparación y evaluación de estudios empíricos sobre bases comunes. La polisemia del concepto de gobernanza plantea un desafío conceptual para la teorización coherente de los desafíos sociales, políticos, ambientales, institucionales y económicos de la acción colectiva a escala local y regional. En lugar de restringir innecesariamente el concepto de gobernanza, quisiéramos definir algunas de sus características comunes mediante la comparación con nociones afines de las ciencias sociales.

\footnotetext{
Geographisches Institut, Universität Heidelberg, Heidelberg, Alemania; glueckler@uni-heidelberg.de Instituto de Geografía, Pontificia Universidad Católica de Chile, Santiago, Chile; jrehner@uc.cl

Geographisches Institut, Universität Heidelberg, Heidelberg, Alemania; handke@uni-heidelberg.de
} 
En general, la gobernanza se refiere a modelos y prácticas de coordinación de la acción colectiva en condiciones de intereses creados, recursos distribuidos y en la búsqueda de objetivos comunes legítimos y aceptados ${ }^{4}$. Aunque es bastante inclusiva, esta definición general se asemeja a una serie de conceptos relacionados en ciencias sociales, tales como gobierno, gestión e instituciones. Todas estas categorías se relacionan con mecanismos que producen y mantienen cierto grado de orden social. Para captar el significado específico de la gobernanza, la ubicamos conceptualmente en un continuo que se expande a lo largo de las dimensiones del tiempo (de la convertibilidad a largo plazo a la convertibilidad a corto plazo) y de la autoridad (de la heteronomía o la interdependencia a la autonomía). Concretamente, concebimos la gobernanza posicionada entre los conceptos de instituciones en un extremo de la coordinación social a largo plazo y de gestión en el otro extremo de la coordinación a corto plazo, donde la toma de decisiones ocurre dentro del ámbito de una autoridad única (organización, Estado).

El concepto de instituciones describe patrones relativamente estables de interacciones sociales basadas en expectativas mutuas legítimas y la aplicación de sanciones sociales (Barley y Tolbert, 1997; Hodgson, 2006; Scott, 2007; Glückler et al., 2018). Las instituciones se refieren a creencias y prácticas legítimas que son compartidas en diferentes grados por la sociedad, garantizan el orden social, ofrecen orientación conductual y facilitan la acción individual en la sociedad. Por lo tanto, es difícil para los individuos y organizaciones cambiar o revocar activamente las instituciones existentes, y si es así, ocurre en períodos más largos. La relativa estabilidad de las instituciones frente a la acción individual ha sido una de las razones por las que el enfoque de la iniciativa empresarial institucional (Lawrence y Phillips, 2004; Leca y Naccache, 2006), que hace hincapié en el poder transformador de las personas emprendedoras, ha evolucionado hasta convertirse en un enfoque de trabajo institucional (Lawrence et al., 2013) que reconoce los límites empíricos de la transformación individual y deliberada de las instituciones.

Mientras que las instituciones se caracterizan por una estabilidad temporal relativa y, por lo general, por un ritmo de transformación lento, el otro extremo del continuo se refiere a las formas actuales de coordinación a corto plazo por medio de la gestión. La gestión describe la administración de una organización, incluyendo la elección de una estrategia y la coordinación de los recursos en la búsqueda del objetivo de la organización. Por consiguiente, la gestión es un modo de coordinación limitado al ámbito de una sola autoridad y legitimado por ella. Los gerentes tienen derecho a tomar decisiones dentro de su jurisdicción organizacional y, por lo tanto, están facultados para asignar recursos, diseñar estructuras y procesos organizacionales, asignar funciones, responsabilidades y resolver conflictos en pos de los objetivos de la organización.

A diferencia de las instituciones a largo plazo y menos dirigibles y de la gestión jerárquicamente controlable a corto plazo, concebimos la gobernanza como la coordinación de las partes interesadas legalmente independientes hacia el cumplimiento de objetivos consensuados. A diferencia de la gestión, la gobernanza va más allá del ámbito de una autoridad única y, por lo tanto, requiere la negociación entre los intereses creados (Rhodes, 1998). Además, la gobernanza difiere de las instituciones sociales en que los procesos de gobernanza se utilizan para abordar

Este acercamiento general al concepto resuena con las concepciones bien establecidas del término, incluyendo, a Jessop (1998), Stoker (1998) o Mayntz (1993: 7) quien de manera similar afirma que "la gobernanza se refiere a la solución de problemas colectivos y a la producción de bienestar público". 
activa y deliberadamente los problemas de acción colectiva y para encontrar soluciones aceptables. Esta amplia comprensión de la controlabilidad interdependiente o 'heterarquía' (Jessop, 1998), que incluye la negociación, la colaboración y la legitimidad, está respaldada en la mayoría de los enfoques de gobernanza.

\section{Gobernanza y geografía (chilena)}

La gobernanza cumple tareas en una amplia variedad de campos y a través de distintas escalas espaciales. Uno de los argumentos clave de este número especial es que la gobernanza no puede concebirse como una categoría sin espacio. Benz y Dose (2010: 33) enfatizan enérgicamente que "si utilizamos el término gobernanza, nos enfrentamos a la tarea de especificar sus características de manera contextualizada e implementar la perspectiva analítica con teorías y métodos apropiados de investigación empírica. Esta es la única manera de obtener declaraciones que son relevantes para la práctica“. Por lo tanto, la geografía y el territorio son elementos cruciales en la concepción, análisis y diseño de la gobernanza (Cerny, 2006; Glückler et. al., de próxima publicación). La gobernanza debe responder a los contextos físicos, sociales, políticos e institucionales específicos de cada espacio, a fin de conciliar los conflictos de intereses por un bien común. La geografía de la gobernanza no solo tiene en cuenta la diversidad y las relaciones entre los espacios, sino también las interdependencias a través de las escalas espaciales. Por lo tanto, hay gobernanza global entre Estados soberanos en cuestiones de derechos humanos y migración, medioambiente y cambio climático, o de la paz. Del mismo modo, identificamos desafíos de gobernanza a escalas espaciales más bajas, en las que las partes buscan encontrar soluciones a los riesgos compartidos colectivamente impuestos por el medioambiente (por ejemplo, sequía, inundaciones, tsunamis, terremotos, etc.) o por la sociedad (por ejemplo, desigualdad social y económica, conflictos de intereses territoriales, etc.). A menudo la gobernanza no puede limitarse a una sola escala, sino que requiere el análisis a varias escalas y de las interrelaciones entre ellas (Storper, 1999).

Debido a su tradicional proximidad a los estudios urbanos más que a la economía o la sociología, la geografía humana latinoamericana, y la investigación chilena en particular, se ha enfocado en la gobernabilidad como un proceso de toma de decisiones sobre temas de planificación del uso de la tierra, especialmente aquellos usos relacionados con las áreas urbanas y la gobernanza urbana. En términos de producción científica, esto se hace evidente, por ejemplo, en el hecho de que la gobernanza es un tema más frecuentemente examinado en la revista de urbanismo EURE que en la Revista de Geografia Norte Grande, aunque tambien se ha publicado un numero especial sobre gobernanza en la revista Geográfica de Valparaiso (Figueroa y Chia 2016). Al inicio del nuevo milenio, la discusión sobre la gobernanza en la geografía latinoamericana se refería más o menos explícitamente a una forma colaborativa, participativa e integradora de planificar y gestionar las ciudades u otros territorios (Arenas, 2008). Tales puntos de vista están arraigados en la tradición de Mayntz y Scharpf (1995), quienes consideran a la gobernanza distinta de la jerarquía del Estado. Esto es coherente con nuestra definición, pero añade un criterio normativo con respecto a la forma en que los diferentes actores deberían interactuar. Esta comprensión caracteriza una visión de gobernanza como nueva forma de coordinación, un modelo idealizado de cómo se deben tomar las decisiones relativas a los territorios (Bustos et al., 2019: 164), específicamente un mecanismo de toma de decisiones que involucra a la sociedad civil (Delamaza-Thayer, 2016; Fernández Tabales et al., 2015), incluyendo el concepto de 'good governance'. En esos casos, aunque 
el criterio de 'interés común' no está siempre claramente definido, se relaciona implícitamente con el bienestar, la prosperidad, la integración social de la ciudad, la conservación del medio ambiente, la sostenibilidad o, en ocasiones, se refiere explícitamente al uso más 'apropiado' del suelo (Vidal y Zamorano, 2016).

Otra corriente de investigación en la geografía latinoamericana se ha centrado en el propio proceso de gobernanza urbana mediante el análisis del ejercicio de poder y las relaciones entre actores a diferentes escalas. Estudios que han examinado cómo las relaciones de poder y las decisiones trascienden y conectan diferentes escalas espaciales (Barton, 2008; Rehner y Pütz, 2008) reflejan una cierta proximidad a la discusión del enfoque de gobernanza multinivel (Parra, 2010; Heinrichs et al., 2009; Klein, 2005). Tales preguntas guían parcialmente contribuciones sobre la relación entre ciudad, gobernabilidad y poder (Yañez et al., 2008) y una colección de trabajos sobre gobernabilidad ambiental (Ruelas Monjardin et al., 2015). Estos enfoques reconocen la naturaleza de la negociación, incluyendo la simultaneidad de la colaboración y el conflicto en las relaciones sociales entre los actores (Torres, 2016).

Los aportes recientes de la geografía latinoamericana y chilena al debate sobre gobernanza señalan críticamente que los enfoques participativos, multiniveles, de buen gobierno y similares son, en primer lugar, medios estratégicos utilizados por actores poderosos para lograr una mayor legitimación de sus decisiones, a menudo motivados por la búsqueda de mayor competitividad (Varela, 2015; Diez-Orueta et al., 2018). Esta visión crítica de la gobernanza como herramienta para aumentar la competitividad territorial ha sido influenciada por el trabajo teórico sobre ciudades y globalización (Sassen, 2007) y por el trabajo de Harvey (1989). Por lo tanto, refleja una crítica al neoliberalismo e identifica la gobernanza urbana como un mecanismo para reemplazar la toma de decisiones liderada por el Estado por asociaciones público-privadas, lo que debilita la influencia del Estado (Bustos et al., 2019; Lukas y Brück 2018). Palabras como 'gobernanza neoliberal' o 'gobernanza empresarial' han surgido para etiquetar esta ideología (De Mattos, 2010 y 2014), y el concepto 'empresarialismo' de Harvey (1989) resuena en la investigación latinoamericana sobre gobernanza urbana (Cuenya y Corral, 2011). Trabajos recientes de esta tradición se alejan de la planificación urbana y abordan cuestiones de geografía económica, por ejemplo, haciendo referencia a la gobernanza neoliberal en la acuicultura (Bustos, 2012) o a la gobernanza financiera mundial (Daher, 2016).

Bustos et al. (2019) y Lukas (2019) discuten de manera sistemática diferentes enfoques de gobernanza. Desde una perspectiva crítica, proponen prestar mayor atención al ejercicio del poder, especialmente para revelar cómo los intereses empresariales hacen uso de la etiqueta de gobernanza y de sus instrumentos para imponer sus propios intereses. En nuestra opinión, el punto crítico de este argumento no es que los actores privados pretendan legitimar sus intereses y decisiones, sino que esas decisiones podrían no reflejar objetivos y consensos comunes. Por lo tanto, este número especial ofrece la posibilidad de discutir una variedad de temas y entendimientos de la gobernanza que enfatizan la idea de negociar objetivos comunes por parte del Estado, de las empresas privadas y otras organizaciones. En el contexto de la gobernanza ambiental, el primer artículo de este número especial se centra en los procesos de negociación y colaboración entre la sociedad civil, el sector privado y los actores estatales de Australia Meridional. Michael Scott (2019) se basa en un análisis cualitativo de los principales actores de la gobernanza del uso de la 
tierra para deconstruir cómo estos actores despliegan técnicas de neutralización para desviar las críticas, gestionar la oposición y justificar nuevos y controvertidos desarrollos costeros amenazados por el aumento del nivel del mar. Explora específicamente cómo estas técnicas se basan en metáforas espaciales tácitas y cómo, finalmente, la legitimidad de los sistemas de planificación del uso de la tierra no se pone en duda. El artículo contribuye así a desmantelar el 'lado oscuro' de la urbanización neoliberal global.

Recientemente, un nuevo enfoque ha ganado cierto protagonismo en América Latina: el concepto de 'buen vivir' (Acosta, 2008), que tiene sus raíces en una epistemología y tradición diferente de producción de saberes. Está ligado a la noción de post-crecimiento, pero basado en una cosmovisión latinoamericana (Unceta, 2014, Arteaga-Cruz, 2017) y, por lo tanto, difiere ideológicamente del concepto de gobernanza. Extrañamente, en algunas publicaciones orientadas a las políticas, el buen vivir se ha fundido en una teleología orientada a los objetivos y la eficiencia (Bravo, 2018; Saavedra, 2014), acercándose más a la gestión (gubernamental) que a la gobernanza, lo que genera cierta confusión en cuanto a las características distintivas de este enfoque y dudas sobre el objetivo común. Para evitar tales contradicciones, el concepto de buen vivir se puede vincular a la gobernanza empleando las reglas de Ostrom para la gobernanza de los bienes comunes, tal como Pamela Mischen, Carl Lipo y Terry Hunt (Mischen et al., 2019) proponen en la segunda contribución en este número. Al aplicar una visión a largo plazo sobre Rapa Nui (Isla de Pascua, Chile), se reconstruyen algunas de las prácticas de gobernanza. Los autores sugieren que muchas de las soluciones actuales de buena gobernanza de los bienes comunes resuenan con la gobernanza pre-europea de la isla. Además, discuten cómo el cambio de las estructuras impuestas por el gobierno central hacia la gobernanza local nativa ha mejorado aspectos importantes, tales como la rendición de cuentas, la inclusión y las necesidades locales.

Otro desafío de gobernanza que es de actualidad para Chile se refiere al riesgo, especialmente en la interfaz entre las amenazas naturales y el hábitat humano. La investigación en este campo estudia el papel de los organismos públicos y los conocimientos técnicos, y a menudo se centra en procesos participativos, por ejemplo, en relación con el riesgo de inundaciones (Usón, et al., 2016), el riesgo sísmico y los deslizamientos de tierra (Castro et al., 2015) o la adaptación al cambio climático (Barton et al., 2015). Además, el programa de investigación Risk Hapitat Megacity ha dedicado parte de sus esfuerzos a analizar la gobernanza del riesgo en Santiago de Chile (Heinrichs et al., 2012). En este contexto, Michael Handke (2019) ofrece un enfoque diferente del trabajo geográfico sobre la gobernanza del riesgo. En el tercer artículo de este número, aborda el riesgo de incendios en la silvicultura chilena. A partir de un estudio de caso cualitativo, examina las prácticas complementarias de gestión de riesgos de las empresas forestales y de las compañías de seguros que tienen como objetivo diversificar y externalizar económicamente el riesgo. Su artículo muestra que el conocimiento geográfico detallado sobre los incendios forestales se descontextualiza explícitamente e incluso se ignora en las prácticas actuales de gestión de riesgos. Se está perdiendo la capacidad de comprender oportunamente las causas y efectos cambiantes de los incendios forestales en Chile, lo que es importante de considerar para un enfoque más amplio de la gobernanza del riesgo en el trabajo. Las responsabilidades sociales no especificadas en la gobernanza del riesgo a escala regional hacen que los incendios forestales se perciban principalmente como efectos externos. 


\section{Gobernanza y redes}

Si la gobernanza se refiere a la coordinación intencionada de la acción colectiva entre partes interesadas interdependientes, entonces el concepto de redes es crucial para comprender cómo funciona la coordinación real. Las personas crean y mantienen redes mediante el intercambio de información, el aprendizaje, la ayuda mutua, el trabajo conjunto y la negociación de decisiones aceptables para un comportamiento legítimo. Es en esta intersección donde la teoría de la gobernanza y la teoría de redes ofrecen un espacio para la fertilización cruzada. La investigación sobre redes no solo ofrece métodos útiles para caracterizar los patrones de relaciones entre conjuntos de actores. También ofrece valiosas teorías sobre cómo las posiciones específicas dentro de una red y las formaciones específicas de redes facilitan ciertos resultados sociales como la innovación, el apoyo social y la solidaridad u otros tipos de resoluciones a los problemas (Kilduff y Tsai, 2003; Knoke, 2012). La investigación reciente sobre las redes sociales pone especial énfasis en dos aspectos (Glückler y Doreian, 2016; Glückler et al., 2017). En primer lugar, niega el determinismo estructural y destaca la relación contingente entre la estructura de la red y los resultados sociales dependiendo del contexto geográfico. Tal contextualidad requiere un conocimiento adicional, sensible al caso y al lugar sobre el entorno físico y el contexto institucional para tener en cuenta el significado de las redes sociales y sus potenciales efectos. En segundo lugar, los métodos de análisis de redes sociales no son fuentes autosuficientes de conocimiento empírico, sino que deben considerarse instrumentos para el desarrollo de teorías sociales.

Desde la perspectiva de la gobernanza, la investigación en economía institucional y sociología económica ha establecido una comprensión de las redes como un modo alternativo de gobernanza frente a los mercados y jerarquías (Powell, 1991). Sin embargo, todavía sabemos poco acerca de qué diseños y procesos de red son adecuados para que las organizaciones faciliten una gobernanza eficaz con propósitos específicos y en contextos particulares. Por lo tanto, solo recientemente ha surgido un campo de investigación sobre la gobernanza de la red (Provan y Kenis, 2008; Maggetti y Gilardi, 2014; Keast, 2016; Berthod et al., 2016). En este número especial, Johannes Glückler (2019) va más allá de considerar a la red como un modo alternativo de gobernanza además de los mercados y las jerarquías. En cambio, en el cuarto artículo de este número especial, reconoce la variación empírica en las formas en que las redes pueden coordinarse para explorar cómo las partes interesadas realmente crean y distribuyen la legitimidad para una gobernanza efectiva de la red. En un intento por superar el dualismo convencional entre los mecanismos formales e informales de gobernanza, Glückler propone el concepto de gobernanza de redes laterales como estructura para la delegación legítima de la toma de decisiones. A partir de la utilización de un enfoque analítico de redes sociales, se compara con casos empíricos de organizaciones de redes de negocios y encuentra patrones reales de legitimidad que difieren significativamente de la autoridad formal de gobierno. Su metodología sirve como herramienta también para la práctica de la gobernanza y ayuda a monitorear y ajustar las redes de gobernanza también en otros contextos.

La distinción entre el proceso de gobernanza y la gobernanza como estructura (el diseño de roles y responsabilidades) pone en juego otros niveles de análisis (Stoker, 1998; Pierre y Peters, 2000). Las tradiciones de la investigación sobre gobernanza también difieren en la forma en que se conciben los actores participantes y los roles que se les atribuyen (Knill y Lehmkuhl, 2002; Peters, 2014). En un extremo, la gobernanza puede ser impulsada como un proceso de 
auto-organización de las partes interesadas (Ostrom et al., 1992; Rhodes, 1996). En el otro extremo, la gobernanza consiste en organizar de manera diferente las relaciones entre el Estado y los actores cívicos (Bell y Hindmoor, 2009). Los papeles del Estado en la gobernanza van desde las alianzas directas con múltiples actores (Osborne, 2000) hasta su acción como 'sombra de la jerarquía' (Heritier y Lehmkuhl, 2008; Chignola, 2014). En la última contribución a este número especial, Lisen Schultz, Simon West y Cláudia Florêncio (Schultz et al., 2019) desarrollan el concepto de gobernanza adaptativa para captar los roles cambiantes de los actores, las prácticas y la política. Sostienen que la capacidad de adaptación es crucial para dar respuestas eficaces de gobernanza a los complejos desafíos de la sostenibilidad. En su evaluación teórica, identifican la necesidad de explorar cómo surge la gobernanza de adaptación en contextos geográficos, y la necesidad de desarrollar relatos de gobernanza de adaptación enraizados en las experiencias cotidianas de aquellos a quienes se les ha asignado hacerla como vacíos fundamentales de investigación. Al proponer un marco analítico que gira en torno a las personas, las prácticas y la política, analizan el surgimiento de la gobernanza adaptativa en la región de la biosfera de Kruger to Canyons, Sudáfrica. Su enfoque sensibiliza a los investigadores para el análisis de la situación y la comprensión de los factores contextuales necesarios para aprender y mejorar la práctica de la gobernanza regional.

Aunque las propuestas seleccionadas en este número contextualizan su análisis empírico en los desafíos específicos de la gobernanza a nivel regional, también reflexionan sobre el impacto de los niveles nacionales o internacionales en las prácticas de la gobernanza regional. En cuanto a la capacidad de resolución de problemas de las prácticas de gobernanza, la teoría tiene que medirse continuamente con los hallazgos empíricos. Para comprender y explicar las peculiaridades de situaciones y casos específicos de gobernanza, se requieren herramientas metodológicas y analíticas adecuadas. Los cinco artículos contribuyen a la comprensión de los enfoques, métodos, procedimientos y prácticas fundamentales de gobernanza. Se emplean diferentes métodos como el análisis de redes sociales (Glückler, 2019), entrevistas personales y análisis cualitativos (Schultz et al., 2019; Scott, 2019, Handke, 2019) y el análisis histórico de documentos y datos (Mischen et al., 2019). Al mismo tiempo, ilustran adaptaciones específicas de modelos de gobernanza más generales en lugares y regiones concretos.

\section{Formación y práctica de gobernanza}

Más allá de la investigación académica, existe una creciente necesidad de habilidades prácticas y expertos calificados para desarrollar, implementar y operar modelos y soluciones de gobernanza para propósitos colectivos específicos y en contextos geográficos particulares. En la práctica, la gobernanza significa más que gobernar y gestionar, ya que implica la capacidad de coordinación de las diferentes organizaciones y sus intereses particulares dentro de un marco institucional específico. Esto requiere diferentes competencias y diálogos entre disciplinas, tradiciones de producción de conocimiento y, lo que es más importante, el desarrollo de capacidades para identificar y negociar objetivos comunes.

En Chile, la Pontificia Universidad Católica de Chile, la Universidad de Chile y la Universidad de Heidelberg (Alemania) han colaborado para establecer un programa de Magister en Ciencias sobre Gobernanza de Riesgo y Recursos. Ubicado en el Centro Heidelberg para América Latina en 
Santiago de Chile, este programa ha atraído desde 2012 a más de 150 estudiantes de disciplinas tan diversas como geografía, sociología, economía, antropología, ingeniería civil, derecho, ingeniería forestal, medicina veterinaria, etc. Además de ser un programa multidisciplinario, también ha desarrollado visibilidad internacional. Estudiantes de 10 países diferentes han estudiado teorías, modelos y métodos de gobernanza para transferir estas habilidades a varios campos de prácticas.

En la búsqueda de un mejor conocimiento de la gobernanza, debe hacerse especial hincapié en el análisis de casos en esta área, para evaluar la utilidad de los modelos, su adecuación y para diseñar soluciones contextuales adecuadas. Además, mediante la recopilación y la comparación constante de casos de gobernanza, se evidencia que los modelos se comportan de manera diferente en distintas situaciones, que desafíos similares pueden afrontarse con modelos diferentes y que los procesos de gobernanza bien diseñados pueden fracasar o tener consecuencias no deseadas.

Chile está expuesto a abundantes riesgos inducidos tanto por la naturaleza como por la sociedad. Por ende, se necesita una conversación académica sobre casos reales de gobernanza, así como conocimientos transformadores y respuestas prácticas para hacer frente a los riesgos ambientales, sociales y económicos, por ejemplo, en relación con el riesgo de tsunamis, inundaciones, sequías e incendios (Martínez y Arranguiz, 2016; Castro et al., 2015; Lagos, 2012; Handke, 2019). Además, Chile enfrenta severos desafíos en materia de recursos naturales, y su relación con aspectos generales del desarrollo, y en asuntos de las herramientas de gestión y gobernanza en campos como la minería, la salmonicultura o la gestión del agua (Rehner y Rodríguez, 2018; Bustos, 2012; Donoso, 2018). Por lo tanto, el país ofrece una plataforma adecuada para seguir elaborando teorías e instrumentos sobre la gobernanza regional de la acción colectiva en América Latina y más allá de sus fronteras.

\section{Conclusión y agenda de investigación}

Con la creciente necesidad de que la geografía aborde los problemas de gobernanza y explore la diversidad de las prácticas de gobernanza local, en este número especial se presentan varias contribuciones en las que se explican con más detalle conceptos alternativos y a menudo complementarios de la gobernanza y se muestra su viabilidad en estudios de casos empíricos. Se presenta aportes de académicos fuera de América Latina con el propósito de hacer más visibles sus propuestas y así nutrir el trabajo sobre gobernanza en la geografía chilena y latinoamericana. Por lo tanto, no pretendemos presentar una visión global de la gobernanza, sino discutir conceptos y técnicas particulares. Los conceptos incluyen la gobernanza de la red (Glückler, 2019), la gobernanza adaptativa (Schultz et al., 2019), la gobernanza del riesgo (Handke, 2019) y la gobernanza ambiental (Scott, 2019), así como la buena gobernanza (Mischen et al., 2019). Proponemos estructurarlas en dos dimensiones, elementos clave de nuestra comprensión de la gobernanza: la orientación a medio plazo y la independencia organizativa de los actores involucrados en un proceso de negociación y coordinación en pos de un objetivo consensuado.

En octubre y noviembre de 2019, Chile ha experimentado las mayores manifestaciones de su historia (algunos la llaman la "Primavera chilena"), pero también un brote imprevisto de violencia. Esto es una expresión de una crisis social profundamente arraigada y se relaciona también con 
un deterioro general de la confianza y la pérdida de legitimidad de una serie de organizaciones. En lo que respecta a muchos temas específicos, la falta de un objetivo común se ha hecho evidente en este contexto. Al tratarse de una crisis institucional, este es uno de los momentos históricamente inusuales en los que algunos arreglos institucionales pueden cambiar con bastante rapidez en respuesta a esta crisis. Una miríada de diferentes actores, individuos, comunidades y organizaciones están reclamando posiciones, negociando y debatiendo sobre objetivos comunes (mayor equidad, terminar con los abusos), aparentemente en búsqueda de la posibilidad de un consenso a pesar de las posiciones profundamente contradictorias. En este contexto, es de particular interés académico analizar cómo reacciona, se adapta y evoluciona la gobernanza, tal como se entiende aquí, incluso en momentos en que el marco institucional se agrieta y cambia repentinamente.

En términos más generales, los elementos centrales de una agenda de investigación geográfica sobre gobernanza tendrán que encontrar respuestas más elaboradas a la pregunta de cómo los actores en entornos territoriales específicos definen, etiquetan y negocian los objetivos comunes y qué mecanismos de gobernanza, estructuras organizativas y procedimientos legítimos son eficaces en esos contextos espaciales e institucionales para lograr objetivos consensuados.

\section{Referencias bibliográficas}

ACOSTA, A. El buen vivir, una oportunidad por construir. Revista Ecuador Debate, 2008, № 75, p. 33-48.

ANSELL, C. \& TORFING, J. (editores). Handbook on Theories of Governance. Cheltenham, UK: Edward Elgar, 2016.

ARENAS, F. Obstáculos para la gobernanza de los espacios metropolitanos chilenos: fragmentación institucional, desarticulación instrumental y atomización social. En: YAÑEZ, G., ORELLANA, A., FIGUEROA, O. y ARENAS, F. (editores). Ciudad, poder, gobernanza. Serie GEOlibros № 9. Santiago: Pontificia Universidad Católica de Chile, 2008, p.67-76.

ARTEAGA-CRUZ, E.L. Buen Vivir (Sumak Kawsay): definiciones, crítica e implicaciones en la planificación del desarrollo en Ecuador. Saúde Debate, 2017, № 41(114), p. 907-919.

BACHE, I. \& FLINDERS, M. (editores). Multi-Level Governance. Oxford: Oxford University Press, 2004.

BARLEY, S. R. \& TOLBERT, P. S. Institutionalization and structuration: Studying the links between action and institution. Organization Studies, 1997, Nº 18 (1), p. 93-117.

BARTON, J. El poder de la gobernanza: el eslabon "perdido" de la sustentabilidad urbana. En: YAÑEZ, G.; ORELLANA, A., FIGUEROA, O. Y ARENAS, F. (ed.) Ciudad, poder, gobernanza. Serie GEOlibros $N^{\circ}$ 9. Santiago: Pontificia Universidad Católica de Chile, 2008, p. 413-430. 
BARTON, J. Climate change adaptive capacity in Santiago de Chile: creating a governance regime for sustainability planning. International Journal of Urban and Regional Research, 2013, № 37 (6), p. 1916-1933.

BARTON, J.R., KRELLENBERG, K., y HARRIS, J. M. Collaborative governance and the challenges of participatory climate change adaptation planning in Santiago de Chile. Climate and Development, 2015, Vol 7, N², p. 175-184.

BELL, S. y HINDMOOR, A. Rethinking Governance. The Centrality of the State in Modern Society. Cambridge: Cambridge University Press, 2009.

BERTHOD, O; GROTHE-HAMMER, M., MÜLLER-SEITZ, G., RAAB, J., SYDOW, J. From high-reliability organizations to high-reliability networks: the dynamics of network governance in the face of emergency. Journal of Public Administration Research and Theory, 2016, N²5, p. 479-511

BEVIR, M. (editor). The Sage Handbook of Governance. London: SAGE, 2010.

BRAVO, O. Indicadores de gobernanza territorial de los objetivos del Plan Nacional del Buen Vivir (PNBV 2013-2017), Ecuador. Civilizar: Ciencias Sociales y Humanas 2018, № 18 (35), p.25-38.

BUSTOS, B. Brote del virus ISA: crisis ambiental y capacidad de la institucionalidad ambiental para manejar el conflicto. Revista EURE - Revista De Estudios Urbano Regionales, 2012, № 38 (115).

BUSTOS, B., LUKAS, M., STAMM, C. \& TORRE, A Neoliberalismo y gobernanza territorial: propuestas y reflexiones a partir del caso de desde Chile. Revista de Geografía Norte Grande, 2019, Nㅜ 73, p. 161-183.

CASTRO, C.P., IBARRA, I.; LUKAS, M.; ORTIZ, J.; SARMIENTO, J.P. Disaster risk construction in the progressive consolidation of informal settlements: Iquique and Puerto Montt (Chile) case studies. International Journal of Disaster Risk Reduction, 2015, Vol. 13, p. 109-127.

CERNY, P. G. Restructuring the State in a Globalizing World: Capital Accumulation, Tangled Hierarchies and the Search for a New Spatio-temporal Fix. Review of International Political Economy, 2006, №13, p. 679-695.

CERRILLO, I. y MARTÍNEZ, A. (editores). La gobernanza hoy: 10 textos de referencia. Madrid: Instituto Nacional de Administración Pública, 2005.

CHHOTRAY, V. y STOKER, G. Governance Theory and Practice: A Cross-Disciplinary Approach. Houndsmill: Palgrave Macmillan, 2009.

CHIGNOLA, S. A la sombra del Estado. Governance, gubernamentalidad, gobierno. Utopía y Praxis Latinoamericana, 2014, № 19, p. 37-51.

CROUCH, C. Capitalist Diversity and Change: Recombinant Governance and Institutional Entrepreneurs. Oxford: Oxford University Press, 2005. 
CUENYA, B. y CORRAL, M. Empresarialismo, economía del suelo y grandes proyectos urbanos: el modelo de Puerto Madero en Buenos Aires. EURE - Revista De Estudios Urbano Regionales, 2011, vol. $37, N^{\circ} 111$, p. $25-45$.

DAHER, A. Externalidades territoriales de la gobernanza financiera global. EURE - Revista De Estudios Urbano Regionales, 2016, № 42 (126).

DELAMAZA, G., y THAYER, L. Percepciones políticas y prácticas de participación como instrumento para la gobernanza de los territorios. Un análisis comparado de escalas territoriales en la macrorregión sur de Chile. Revista EURE - Revista De Estudios Urbano Regionales, 2016, № 42 (127).

DE MATTOS, C. Una nueva geografía latinoamericana en el tránsito de la planificación a la gobernanza, del desarrollo al crecimiento. Revista EURE - Revista De Estudios Urbano Regionales, 2010, $N^{\circ} 36$ (108), p.167-179.

DE MATTOS, C. Gobernanza neoliberal, financiarización y metamorfosis urbana en el siglo XXI, 2014.

DIAZ-ORUETA, F., LOURÉS, M., y PRADEL-MIQUEL, M. Transformando los modelos de crecimiento y cohesión: cambios en la gobernanza de Barcelona y Madrid. EURE - Revista De Estudios Urbano Regionales, 2018, № 44(131).

DONOSO, G. (Editor ) Water Policy in Chile. Global Issues in Water Policy 21. Heidelberg: Springer. 2018.

FEIOCK, R. C. Rational choice and regional governance. Journal of Urban Affairs, 2007, N² 29 (1), p. 47-63.

FERNANDEZ TABALES, A. et al. Gobernanza territorial y gestión de espacios turísticos en contextos de fuerte presión inmobiliaria: Análisis de buenas prácticas locales en la costa de Cádiz (España). Revista de Geografía Norte Grande, 2015, № 60, p. 173-194.

FIGUEROA, R. y CHIA, E. Introduccion al número especial. Gobernanza territorial, conflictos y aprendizajes. Revista Geográfica de Valparaiso, 2016, No 53, p. 1-6.

GARCIA, M. Citizenship practices and urban governance in European cities. Urban Studies, 2006, $N^{\circ} 43(4)$, p. $745-765$.

GEREFFI, G., HUMPHREY, J. y STURGEON, T. The governance of global value chains. Review of International Political Economy, 2005, N 12, p. 78-104.

GLÜCKLER, J. Gobernanza lateral de redes: Legitimidad y delegación relacional de la autoridad decisoria. Revista de Geografía Norte Grande, 2019, N 74, p. 93-115. 
GLÜCKLER, J. y DOREIAN, P. Editorial: Social network analysis and economic geography - positional, evolutionary and multi-level approaches. Journal of Economic Geography, 2016, № 16 (6), p. 1123-1134.

GLÜCKLER, J., HERRIGEL, G. y HANDKE, M. (editores). Knowledge for Governance. Knowledge and Space, vol. 15. Heidelberg: Springer, forthcoming

GLÜCKLER, J., LAZEGA, E. y HAMMER, I. (editores). Knowledge and Networks. Knowledge and Space Series, vol. 11. Heidelberg: Springer, 2017.

GLÜCKLER, J., SUDDABY, R. y LENZ, R. (editores). Knowledge and Institutions. Knowledge and Space, vol. 13. Heidelberg: Springer, 2018.

HANDKE, M. Valorización del riesgo de incendios forestales con conocimiento geográfico: Los desafíos de la gobernanza. Revista de Geografía Norte Grande, 2019, ํo 74, p. 65-91.

HARVEY, D. From Managerialism to Entrepreneurialism: The Transformation in Urban Governance in Late Capitalism, Geografiska Annaler: Series B, Human Geography, 1989, № 71(1), p. 3-17.

HEINRICHS, D.; NUISSL, H.; RODRIGUEZ SEEGER, C. Dispersión urbana y nuevos desafíos para la gobernanza (metropolitana) en América Latina: el caso de Santiago de Chile. EURE - Revista De Estudios Urbano Regionales, 2009, №35(104), p. 29-46.

HEINRICHS, D.; KRELLENBERG, K.; HANSJÜRGENS, B.; MARTINEZ, F. Risk Habitat Megacity. Heidelberg: Springer, 2012.

HERITIER, A. y LEHMKUHL, D. Introduction. The shadow of hierarchy and new modes of governance. Journal of Public Policy, 2008, №. 28, p. 1-17.

HIRST, P. y THOMPSON, G. Globalization in Question. The International Economy and the Possibilities of Governance. Cambridge, Malden, MA: Polity Press, 1996.

HODGSON, G. M. What are institutions? Journal of Economic Issues, 2006, № 40, p. 1-25.

HUGHES, A. Geographies of exchange and circulation: transnational trade and governance. Progress in Human Geography, 2006, № 30 (5), p. 635-643.

JESSOP, B. The rise of governance and the risks of failure: the case of economic development. International Social Science Journal, 1998, 155.

KEAST, R. Network governance. En: ANSELL, C. y TORFING, J. (editores). Handbook on Theories of Governance. Cheltenham, UK: Edward Elgar, 2016, p. 442-453.

KILDUFF, M. y TSAI, W. Social Networks and Organizations. London: Sage, 2003. 
KLEIN, J. -L. Iniciativa local y desarrollo: respuesta social a la globalización neoliberal. EURE - Revista De Estudios Urbano Regionales, 2005, № 31 (94), p. 25-39.

KNILL, C.; LEHMKUHL, D. Private actors and the state: internationalization and changing patterns of governance. Governance: An International Journal of Policy, Administration, and Institutions, $2002, N^{\circ} 15$, p. 41-63.

KNOKE, D. Economic Networks. Cambridge, UK: Polity Press, 2012.

LAGOS, M. Zonificación del riesgo de tsunami en el centro-sur de Chile. Revista de Geografía Norte Grande 2012, № 53, p. 7-21.

LAWRENCE, T. B. \& PHILLIPS, N. From Moby Dick to Free Willy: macro-cultural discourse and institutional entrepreneurship in emerging institutional fields. Organization, 2004, No 11 (5), p. 689-711.

LAWRENCE, T. B., LECA, B. y ZIMMER, T. B. Institutional work: Current research, new directions and overlooked issues. Organization Studies, 2013, 34, 1023-1033.

LECA, B. y NACCACHE, P. A critical realist approach to institutional entrepreneurship. Organization, 2006, No 13 (5), p. 627-651.

LEVI-FAUR, D. (editor). Oxford Handbook of Governance. Oxford: Oxford University Press, 2012.

LIVERMAN, D. Who governs, at what scale and at what price? Geography, environmental governance, and the commodification of nature. Annals of the Association of American Geographers, 2004, No 94, p. 734-738.

LUKAS, M. Urban Governance. En: ORUM, A. (ed.). The Wiley-Blackwell Encyclopedia of Urban and Regional Studies. Hoboken, NJ: Wiley-Blackwell, 2019 (doi:10.1002/9781118568446.eurs0366).

LUKAS, M. y BRÜCK, A. Urban Policy Mobilities und globale Produktionsnetzwerke. Städtische Planung in Chile als Legitimationsinstanz extraktiver Industrien. Sub/urban $N^{\circ} 6,2 / 3,2018$, p. 69-90.

MAGGETTI, M. y GILARDI, F. Network governance and the domestic adoption of soft rules. Journal of European Public Policy, 2014, N² 21 (9), p. 1293-1310.

MAYNTZ, R. y SCHARPF, F. (editores). Gesellschaftliche Selbstregelung und politische Steuerung. Frankfurt/ Main: Campus, 1995.

MAYNTZ, R. Governing failures and the problem of governability: Some comments on a theoretical paradigm. En: J. KOOIMAN (editor): Modern Governance. New Government - Society Interactions. London: Sage, 9-20, 2003. 
MAYNTZ, R. Nuevos desafíos de la teoría de la gobernanza. En: A. CERRILLO y I. MARTÍNEZ (editores). La obernanza hoy: 10 textos de referencia. Madrid: Instituto Nacional de Administración Pública, 83-98, 2005.

MARTINEZ, C. y ARANGUIZ, R. Riesgo de tsunami y planificación resiliente de la costa chilena: La localidad de Boca Sur, San Pedro de la Paz ( $37^{\circ} \mathrm{S}$ ). Revista de Geografia Norte Grande, 2016, N64, p. 33-54.

MISCHEN, P. A., LIPO, C. P. y HUNT, T. L. Good governance of the commons of Rapa Nui (Easter Island, Chile): present and past. Revista de Geografía Norte Grande, 2019, Nº 74, p. 39-63.

MONTERO, S. y CHAPPLE, K. (editores). Fragile Governance and Local Economic Development: Theory and Evidence from Peripheral Regions in Latin America. New York: Routledge, 2019.

MOULAERT, F., PARRA, C., y SWYNGEDOUW, E. Ciudades, barrios y gobernanza multiescalar en la Europa urbana. EURE - Revista De Estudios Urbano Regionales, 2014, № 40 (119).

NUISSL, H., HÖHNKE, C., LUKAS, M., DURÁN, G., SEEGER, C (editores.) Megacity Governance: Concepts and Challenges. Berlin: Springer, 2012.

OSBORNE, S. Understanding public-private partnerships in international perspective. London: Routledge, 2000.

OSTROM, E. Governing the Commons: The Evolution of Institutions for Collective Action. Cambridge, UK: Cambridge University Press, 1990.

OSTROM, E. Understanding Institutional Diversity. Princeton: Princeton University Press, 2005.

OSTROM, E., WALKER, J. y GARDNER, R. Covenants with and without a sword. Self-governance is possible. American Political Science Review, 1992, № 86, p. 404-417.

PARRA, C. Sustainability and multi-level governance of territories classified as protected areas in France: the Morvan regional park case, Journal of Environmental Planning and Management, 2010, $N^{\circ}$ 53:4, p. 491-509.

PETERS, B. G. Is governance for everybody? Policy and Society, 2014, № 33, p. 301-306.

PIERRE, J. \& PETERS, B. G. Governance, Politics, and the State. London: MacMillan Press, 2000.

POWELL, W. Neither market nor hierarchy: Network forms of organization. En: G. THOMPSON, J. FRANCES, R. LEVACIC y J. MITCHELL (editores). Markets, Hierarchies and Networks. London: Sage, 1991(pp. 265-276)

PROVAN, K. G. \& KENIS, P. Modes of network governance: Structure, management, and effectiveness. Journal of Public Administration Research \& Theory, 2008, № 18, p. 229-252. 
REHNER, J.; PÜTZ, M. Gobernanza urbana, ordenamiento territorial y actos de poder en proyectos urbanos conflictivos en Munich / Alemania. En: G. YÁÑEZ, A. ORELLANA, O. FIGUEROA, F. ARENAS (editores): Ciudad, Poder, Gobernanza. Santiago de Chile. Serie GeoLibros No 9, 2008, p. 291-317.

REHNER, J. y RODRIGUEZ, S. Cities built on copper - the impact of mining exports, wages and financial liquidity on urban economies in Chile. Resources Policy, 2018.

RHODES, R. The new governance: governing without government. Political Studies, 1996, $N^{\circ} 44$, p. 652-667.

RUELAS MONJARDIN, L.C., TRAVIESO-BELLO, A.C. y SÁNCHEZ SÁNCHEZ, O. (editores). Gobernanza ambiental: teoría y práctica para la conservación y uso sustentable de los recursos. Veracruz: El Colegio de Veracruz, 2015.

SAAVEDRA BUSTOS, C.A. La gobernanza democrática, la gobernabilidad y el buen vivir en la provincia de Esmeraldas (Ecuador), desde la perspectiva sociocultural, ética y política. Administracion \& Desarrollo, 2014, № 43 (59), p. 53-62.

SASSEN, S. El reposicionamiento de las ciudades y regiones urbanas en una economía global: ampliando las opciones de políticas y gobernanza. EURE - Revista De Estudios Urbano Regionales, 2007, 33 (100) 9-34.

SCHULTZ, L., WEST, S. y FLORÊNCIO, C. Adaptive governance: People, practices, politics in a UNESCO biosphere reserve. Revista de Geografía Norte Grande, 2019, № 74, p. 117-138.

SCOTT, M. The 'dark side' of land use governance: Spatiotemporal narratives and the neutralizing of environmental risk. Revista de Geografía Norte Grande, 2019, № 74, p. 21-37.

SCOTT, W. R. Institutions and Organizations. Thousand Oaks: Sage, 2007.

STOKER, G. Governance as theory: five propositions. International Social Science Journal, 1998, $N^{\circ} 50$, p. 17-28.

STORPER, M. y HARRISON, B. Flexibility, hierarchy and regional development: The changing structure of industrial production systems and their forms of governance in the 1990s. Research Policy, $1991, N^{\circ} 20$ (5), p. 407-422.

STORPER, M. The resurgence of regional economics ten years later: The region as a nexus of untraded interdependencies. En: J. BRYSON, N. HENRY, D. KEEBLE y R. MARTIN (editores): The Economic Geography Reader: Producing and Consuming Global Capitalism. Chichester, New York: John Wiley \& Sons, 207-215, 1999.

SWYNGEDOUW, E. Governance innovation and the citizen: The Janus face of governance-beyond-the-state. Urban Studies, 2005, N²2(11). 
UNCETA, K. Poscrecimiento, desmercantilización y «buen vivir». Nueva Sociedad, 2014, № 252, p. 136-153.

USÓN, T.J., KLONNER, C. y HÖFLE, B. Using participatory geographic approaches for urban flood risk in Santiago de Chile: Insights from a governance analysis. Environmental Science \& Policy 2016. $N^{\circ} 66$, p. 62-72

VARELA, E. Nuevos roles de los gobiernos locales en la implementación de políticas públicas. Gobernabilidad territorial y competitividad global. EURE - Revista De Estudios Urbano Regionales, 2015, 41(123).

VIDAL BRAVO, A. y ZAMORANO CARREÑO, C. De la participación a la gobernanza. Conflictos y aprendizajes desde la escala local a la regional. El caso del área norte de la comuna de Quilpué. Revista Geográfica Valparaíso, 2016, № 53, p. 72- 91.

WILLIAMSON, O. E. The economics of governance. American Economic Review, 2005, № 95, p. $1-18$.

YAÑEZ WARNER, G., et al. (editores). Ciudad, Poder, Gobernanza. Santiago de Chile: Instituto de Geografía, Pontificia Universidad Católica de Chile, Serie GEOlibros N 9, 2008. 